LA-UR-97-- 2564

Approved for public release; distribution is unlimited
$1962 ? 1907$

OSTI

Title: Transport Processes in Space Plasmas

Author(s):

J. Birn, R. C. Elphic, W. C. Feldman, S. P. Gary, and

J. T. Gosling, NIS-1

R. J. Mason, M. E. Jones, and D. Winske, X-PA

V. Chow, H. Karimabadi, D. Krauss-Varban, M. E. McKean,

D. A. Mendis, N. Omidi, U. Samilo, and M. Rosenberg,

U.C.-San Diego

M. Kelley, N. Ohtani, M. Oppenheim, C. Seyler, and

R. N. Sudan, Cornell U.

V. Angelopoulos, P. Convery, C. F. Kennel, H. Kuo, G. Le, G. M. Lindsay, S. Lochbiler, J. J. Luhman, J. Newbury, C. T. Russell, and D. Schriver, U.C.-Los Angeles

H. Rahman and G. Yur, U.C.-Riverside

Submitted to:

DOE Office of Scientific and Technical Information (OSTI)

Los Alamos National Laboratory, an affirmative action/equal opportunity employer, is operated by the University of Calfomia for the U.S. Department of Energy under contract W-7405-ENG-36. By acceptance of this article, the publisher recognizes that the U.S. Government retains a nonexchusive, royaltyfree license to publish or reproduce the published form of this contribution, or to allow others to do so, for U.S. Government purposes. Los Alamos National Laboratory requests that the publisher identify this article as work performed under the auspices of the U.S. Department of Energy. Los Alamos National Laboratory stronghy supports academic freedom and a researcher's right to publish; as an institution, however, the Laboratory does not endorse the viewpoint of a publication or guarantee its technical correctness. 


\section{DISCLAMIER}

Portions of this document may be illegible in electronic image products. Images are produced from the best available original document. 


\title{
Transport Processes in Space Plasmas
}

\author{
Joachim Birn, Richard C. Elphic, William C. Feldman, \\ S. Peter Gary and John T. Gosling \\ Nonproliferation and International Security Division \\ Los Alamos National Laboratory
}

Ron J. Mason, Michael E. Jones and Dan Winske

Applied Theoretical and Computational Physics Division

Los Alamos National Laboratory
Vivian Chow, Homayoun Karimabadi, Dietmar Krauss-Varban, Michael E. McKean, D. Asoka Mendis, Nojan Omidi, U. Samilo, and Marlene Rosenberg University of California - San Diego Mike Kelley, Niels Ohtani, Meers Oppenheim, Charles Seyler and Ravi N. Sudan
Cornell University

\author{
Vassilis Angelopoulos, Patrick Convery, Charles F. Kennel, Han Kuo, \\ Guan Le, Gretchen M. Lindsay, S. Lochbiler, Janet J. Luhman, \\ Jennifer Newbury, Christopher T. Russell and David Schriver \\ University of California - Los Angeles \\ Hafiz-ur-Rahman and Gung Yur \\ University of California - Riverside
}

\begin{abstract}
This is the final report of a three-year, Laboratory Directed Research and Development (LDRD) project at the Los Alamos National Laboratory (LANL). The project represents a comprehensive research effort to study plasma and field transport processes relevant for solar-terrestrial interaction, involving the solar wind and imbedded magnetic field and plasma structures, the bow shock of the Earth's magnetosphere and associated waves, the Earth's magnetopause with imbedded flux rope structures and their connection with the Earth, plasma flow in the Earth's magnetotail, and ionospheric beam/wave interactions. The focus of the work was on the interaction between plasma and magnetic and electric fields in the regions where different plasma populations exist adjacent to or superposed on each other. These are the regions of particularly dynamic plasma behavior, important for plasma and energy transport and rapid energy releases. The research addressed questions about how this interaction takes place, what waves, instabilities, and particle/field interactions are involved, how the penetration of plasma and energy through characteristic boundaries takes place, and how the characteristic properties of the plasmas and fields of the different populations influence each other on different spatial and temporal scales. These topics were investigated through combining efforts in the analysis of plasma and field data obtained through space missions with theory and computer simulations of the plasma behavior.
\end{abstract}

\footnotetext{
- Principal Investigator, e-mail: jbirn @ lanl.gov
} 


\section{Background and Research Objectives}

Space plasma physics covers the physics of ionized gases in the interplanetary region. It includes the magnetospheres of the planets, that is, the regions above the planets' surfaces that are governed by their magnetic fields. The prime region of interest is the Earth's magnetosphere, shaped through its interaction with the surrounding medium of charged particles ejected from the sun, the solar wind. The magnetosphere occupies a region of roughly 10 to 15 earth radii above the surface on the dayside and extends into a long tail, like a comet's tail, of hundreds of earth radii length on the nightside. The interest in its structure, its charged particle population (plasma), and the dynamic processes operating within it stems not only from the fact that these processes cause the spectacular auroras (polar lights), perturb the earth's magnetic field, and affect satellite measurements and operations, but also from the fact that the underlying processes are characteristic of plasma behavior in many other areas such as laboratory plasma confinement experiments as well as solar flares and astrophysical phenomena.

Unlike astrophysical objects, the magnetosphere and the solar wind are accessible, through satellites, to direct measurements of their magnetic and electric fields and particle populations. The satellites carrying the scientific instruments are tiny in comparison to the characteristic dimensions of the particle and field structures within these regions. The measurements are therefore far less disturbing and more detailed than those obtained in laboratory experiments. The detailed knowledge of particle distributions and fields in the magnetosphere and the solar wind, which may vary on a wide range of temporal and spatial scales, hence provides an ideal testing ground for theoretical studies and high performance computer modeling of plasma behavior.

The research of this project concerned primarily the regions where one plasma population interacts with a different adjacent population or with some different surrounding medium. These are the regions of particularly dynamic plasma behavior, where plasma and energy transport and dynamic energy release occur. The research focused on questions concerning how this interaction takes place, what waves, instabilities, and particle/field interactions are involved, how the penetration of plasma and energy through characteristic boundaries takes place, and how the characteristic properties of the plasmas and fields of the different populations influence each other on different spatial and temporal scales. The scientific applications include (1) the interaction of plasma blobs ejected from the sun ("coronal mass ejections") with the surrounding solar-wind plasma and fields, (2) wave and particle interactions at the bow shock of the Earth's magnetosphere and at the magnetopause, which separates the magnetosphere from the solar wind, (3) the influence of the 
solar wind on the overall magnetospheric structure, (4) the properties of particle acceleration, transport, and plasma flow bursts within the magnetospheric tail, and (5) electron and ion heating through instabilities in the ionosphere and the magnetosphere.

\section{Importance to LANL's Science and Technology Base and National R\&D Needs}

In order to understand more fully the solar-terrestrial interactions and their implications, a coordinated research effort in data interpretation and computer modeling is necessary. The investigations were based on two outstanding Laboratory competencies and capabilities. The first capability is the Laboratory's unique expertise from its thirty-year program for the development, flight, and analysis of data from instruments to measure plasma and energetic charged particles in near-Earth space. The second one is the unique Laboratory capability in high performance computing and the development and application of plasma simulation codes. An important aspect was not only the utilization of these unique capabilities, but also the further enhancement of the capabilites through the connections with university expertise and resources. The improvement and continuing development of the plasma simulation codes is an obvious part of the interactive application of the codes to the space plasma transport and wave-particle interaction problems. The experimental and data analysis part of the research, however, also enhanced the Laboratory's expertise, not only in improving the handling and analyzing of the existing data sets, but also in giving strong impulses to the further development of instrumentation of future space experiments.

Los Alamos capabilities were ideally complemented by University of California (UC) competencies and resources: Laboratory plasma and energetic particle data by UCLos Angeles (UCLA) magnetic field data; Laboratory magnetotail modeling codes and expertise by the UC-Riverside (UCR) magnetospheric simulation laboratory experiment; and Laboratory and UC-San Diego (UCSD) sharing highly collaborative computer simulation studies of magnetospheric boundary layers. By exchanging different types of satellite data, by using Laboratory computer simulations to model UC experiments, and by exchange visits of students, postdocs and staff, the Laboratory and UC worked together to produce space plasma research that is more complete and of higher quality than if each institution had worked separately. 


\section{Scientific Approach and Accomplishments}

\section{Study of Interplanetary Structures Associated with Coronal Mass Ejections}

Coronal mass ejections, CMEs, are known to be the primary sources of major interplanetary disturbances and large geomagnetic storms, but the details of their origin and structure are still subjects of active investigation. Los Alamos research has long played a dominant role in discovering, identifying, and analyzing CMEs. One part of the present investigation was aimed at obtaining information on the three-dimensional (3-D) shapes of CMEs by studying the nature of solar wind flow deflections produced by fast CMEs plowing their way outward through the solar wind. Pioneer Venus Orbiter (PVO) solar wind plasma and magnetic field data were used to study such deflections. A surprising result of this study is that CMEs are more effective than corotating stream interaction regions in producing north-south deflections in the flow.

It is well known that the size of a geomagnetic storm is directly related to the solar wind flow speed and the strength and orientation of the interplanetary magnetic field associated with a CME-driven disturbance. An effective storm forecasting system thus requires a means of determining at least these properties for earthward-directed CME events. Although the space-based quadrature concept has been repeatedly suggested, its merit as a means of predicting geomagnetic disturbances has never been evaluated in any detail. The current study took advantage of a prototype provided by the combination of coronagraph data obtained from near-Earth orbit and solar wind data obtained $90^{\circ}$ off the Sun-Earth line. CMEs that occur when the PVO or the Helios spacecraft is above the solar limb, as observed from Earth, were used to determine how well we can predict speed and field enhancements in solar wind disturbances from coronagraph observations of the CMEs causing the disturbances.

Several particularly well-defined cases of paired CME and interplanetary disturbance observations were identified and analyzed. The results of this first survey showed how the disturbance speed near $1 \mathrm{AU}$ relates to the CME speed observed in the coronagraph images. Slow CMEs get accelerated up to at least the minimum speed of the normal solar wind, while fast CMEs decelerate as they interact with the ambient plasma ahead and behind. Our survey clearly shows that the fastest CMEs in the corona produced the strongest enhancements in the interplanetary magnetic field, as expected from both previous observational studies and theoretical predictions. 


\section{Structure of the Magnetopause}

The Earth's magnetopause separates the solar wind plasma, which has been slowed and heated by the bow shock and lies on open field lines, from hotter plasma confined to the magnetosphere on magnetic field lines that are connected to the Earth. An ultimate goal of magnetospheric space physics is to understand and predict the flow and conversion of energy from the solar wind into and through the magnetosphere and its ultimate effect on the Earth. To develop this predictive capability, an understanding of the magnetopause and its underlying physics is essential. This involves a detailed examination of its internal spatial and temporal scales, and how the magnetopause is affected by external stimuli (e.g., shock generated waves or variations in the solar wind conditions).

In the magnetohydrodynamic (MHD) limit the magnetopause can either be a simple tangential discontinuity (TD) during northward interplanetary magnetic field (IMF) or a combination of a rotational discontinuity (RD) and other waves or discontinuities (such as a slow shock and/or a contact discontinuity) during southward IMF. Spacecraft observations, however, have shown that even during northward IMF the magnetopause may have a much more complex structure consisting of several distinct plasma layers whose widths are typically on the order of several ion gyroradii. Similarly, except for the presence of RDs, the other expected discontinuities (e.g., slow shocks) have not been identified during southward IMF. This complex structure and also the observed short scale lengths suggest strongly that the understanding of the magnetopause is fundamentally a kinetic problem which should include the dynamic formation of multiple discontinuities and layers. While the kinetic structure of isolated RDs and TDs have been studied in the past, the overall structure of the magnetopause had not been studied in a kinetic approach. To address this shortcoming, a new approach was used where multiple discontinuities are formed in a dynamic fashion in the simulations. Using this approach, the validity of the MHD magnetopause models was investigated.

A comparison between the observations and the simulation results shows considerable resemblance. Both the observed density and magnetic field profiles show oscillations in the magnetosheath with a clear anticorrelation. This property of the waves suggests that they are either slow magnetosonic or mirror modes. A recent study by Song et al. (1994) has shown that the waves have characteristics that distinguish them from the well known mirror mode in the magnetosheath. They have a larger wavelength and, more importantly, a finite phase velocity in the plasma rest frame. Song et al. have thus concluded that these waves are slow magnetosonic waves. The present extensive study of these waves has shown that the excited mirror waves have wavelengths and amplitudes that are considerably smaller than the waves seen in the simulations. It has also established that the waves near 
the magnetopause have a finite phase velocity in the plasma rest frame. This is all in agreement with the observations of Song et al. and supports their conclusion that these waves are not mirror modes. In contrast, one-dimensional (1-D) two-fluid simulations have demonstrated the absence of mirror and Alfven waves in the fluid limit, as well as the absence of a jump in the density downstream of the intermediate shock. These results illustrate that the simple MHD picture of the magnetopause is greatly increased in complexity by kinetic effects.

Local two-dimensional (2-D) simulations of the magnetopause, concerning the interaction between the solar wind and the magnetospheric plasmas, resulted in the formation of a fast shock (representing the bow shock) and a region containing sharp and smooth gradients which collectively is referred to as the magnetopause. Some of the boundaries found in this region correspond to a classical discontinuity (such as a rotational discontinuity or an intermediate shock) while others don't. Most notable is the presence of the magnetic boundary across which the magnetic field strength changes abruptly but other plasma parameters do not (Omidi and Winske, 1995).

Another aspect of our investigation has been with regard to plasma diffusion due to surface waves at the magnetopause. In the study by Winske and Omidi (1995), we showed that the magnetic boundary is unstable to the generation of surface waves which correspond to the long-wavelength limit of the lower hybrid instability. Analysis of the simulation data has shown that these waves do lead to particle diffusion across the magnetopause. However, the level of plasma transport is not sufficient to account for the presence of the low-latitude boundary layer. As such, we have concluded that these surface waves are not a primary source of plasma transport at the magnetopause. Similarly, by studying the ion distribution functions, we have shown that these waves do not lead to any appreciable ion heating. Despite all of this, these low-frequency drift waves may be responsible for some of the density structures observed at the boundary layer by Song et al. (1994). In these observations, the change from magnetosheath to magnetospheric densities did not take place in a smooth manner, but rather took place through a number of sharp jumps. Although our 1-D simulations could explain many of the magnetopause features observed by Song et al. (1994), the simulated density profile within the boundary layer was smooth without any sharp gradients. However, in the 2-D simulations, because of the surface waves, this transition is not smooth and occurs in a discontinuous manner. This behavior may explain the observations by Song et al.

The major conclusions reached in the above studies can be summarized as follows. The interaction of the mirror waves with the magnetic boundary at the magnetopause modifies their properties in a number of ways: (1) it leads to their amplification, (2) it causes 
them to have a finite frequency in the plasma rest frame, (3) it changes their direction of the propagation so that their wave vector is along the magnetopause normal. As a consequence, these waves can be used as means of diagnosing the rate of plasma entry into the magnetosphere. The magnetic boundary is also found to be unstable to the generation of surface waves. These waves are the long wavelength limit of the lower hybrid drift waves. We have found that while the surface waves lead to particle diffusion across the magnetopause, the degree of transport is not sufficient to account for the formation of the low-latitude boundary layer. These waves also result in density structures within the boundary layer, which may account for observations by Song et al.

\section{Properties of Magnetopause Flux Transfer Events}

The interaction between the solar wind and the magnetosphere at the magnetopause commonly takes place in localized regions where interconnected magnetic flux tubes are formed, called flux transfer events (FTEs). The signature of FTEs is a strong component of the magnetic field along the nominal normal to the magnetospheric boundary with a bipolar perturbation, outward, then inward or vice versa. They can be observed both in the magnetosheath (that is, the solar wind plasma surrounding the magnetosphere after passing through the bow shock) and in the magnetosphere near the magnetopause. The original suggestion for the appearance of FTEs was that they represented the results of a temporary increase in the reconnection rate between magnetospheric and magnetosheath field lines. The resulting reconnected flux tube would go from the solar wind into the magnetosphere and down to the ionosphere. The observed bipolar perturbations are the signatures of moving reconnected flux tubes. Early statistical studies revealed that FTEs occur preferentially during southward IMF and the polarity of the bipolar signatures is ordered by the geomagnetic equator in a manner consistent with the global picture of reconnected flux tubes moving away from the equatorial region.

FTEs occur with a nearly periodic rate but not precisely periodic. The average or median spacing is about 8 minutes but covers a very wide range. It is not evident what controls that spacing. We have looked at solar wind dynamic pressure and Mach number and found no correlation with the FTE spacing. In contrast to a suggestion by Lockwood and Wild (1994), we found that the IMF is usually steady prior to the occurrence of an FTE. Hence, FTEs seem not to be driven by fluctuations of the IMF.

FTEs are clearly associated with a southward component of the IMF and thus are most certainly reconnection phenomena. This fact, which is true for both magnetosheath and magnetospheric FTEs, was further demonstrated by measuring the rates of occurrence of FTEs in the magnetosheath and magnetosphere as a function of IMF clock angle. We 
found that FTEs disappear for northward IMF but occur almost uniformly with angle once the MF is southward. The difference in rates between the magnetosphere and magnetosheath can be attributed to the smaller magnetospheric effect of FTEs. In addition, we found that increasing solar wind Mach number reduces slightly the occurrence rate.

Even though their properties are similar, the number of magnetospheric FTEs is about half of that of magnetosheath FTEs. This difference may result from smaller bipolar signatures of magnetospheric FTEs than those of magnetosheath FTEs, due to the fact that the field strength is typically smaller in the magnetosheath, resulting in smaller magnetic tension force and consequently larger distortion of the magnetosheath field lines. Finally, we found that both the magnetosheath and magnetospheric FTEs have the same polarity variations with latitudes as demonstrated by previous studies. This polarity reversal around the equator is consistent with the moving reconnected flux tube model but not consistent with a boundary wave model. We also found that the polarity of FTEs is generally the same on either side of the magnetopause (about $90 \%$ of the cases).

Ion distribution functions reveal that the ions in the magnetospheric FTE distribution behave just as transmitted ions do in reconnection events of accelerated flow at the magnetopause. The peaks of both distributions are about two magnetosheath Alfvén speeds away from the magnetosheath distribution, as expected from reconnection models. Flux transfer events hence appear to be manifestations of transient reconnection of the magnetospheric and magnetosheath magnetic fields. However, the mechanism by which this occurs is presently quite uncertain. Global simulations, both laboratory and computer, suggest that FTEs arise from high latitude reconnection in both the north and south, forming a rope that may not be connected to the Earth. Ionospheric observations, on the other hand, show features that researchers have suggested are caused by FTEs. If true, the FTE would have to be connected to the ionosphere. The Los Alamos Fast Plasma Experiment observations on the ISEE spacecraft were able to resolve the dilemma through the detection of ionospheric plasma. Previous studies of FTEs have shown, consistent with both models, that FTEs are a mixture of magnetosheath and magnetospheric plasma but no one has yet searched for ionospheric plasma in FTEs. We have successfully extended this technique to the boundary layer, which shows evidence of ionospheric plasma and therefore connection of field lines to the Earth.

Electric field data and the electron moments from the Vector Electron Spectrometer (VES) were used to determine if FTEs propagate upward or downward according to their polarity. By examining the $\mathbf{E} \times \mathbf{B}$ drift velocity, we found that "standard" FTEs move 
northward and "reverse" FTEs move southward. The result again suggested that reconnection is the source of FTEs.

\section{Three-Dimensional Magnetotail Structure}

One of the major restrictions of our understanding of magnetosphere properties from space data is the fact that input parameters (typically the solar wind parameters) cannot be controlled, often they are coupled, so that their relative importance cannot be separated, or are not measured at all. In addition, simultaneous measurements by several satellites in different locations are rare and restricted by their orbits. Statistical analyses of average properties, on the other hand, can hardly ever be made under exactly the same external conditions. Laboratory experiments simulating the interaction of a flowing magnetized plasma with a magnetic dipole and computer simulations of such interaction can therefore provide a very valuable complement to space data analysis.

A primary objective of the laboratory simulation experiments together with accompanying theory and computer simulations was the investigation of the global structure of the simulated magnetosphere and its imbedded boundaries for different polarities and magnitudes of the applied solar wind magnetic field. The main goal was to find how the characteristic scales and perhaps the existence of characteristic boundary layers depend on the solar wind parameters. Our study built upon our previous investigation of the magnetotail (Birn et al.), which explored this region through models and magnetic field measurements in the simulated magnetosphere. These measurements were now complemented by measurements of plasma flow and temperature. The new results demonstrated, for the first time in a laboratory, the presence of a magnetotail plasma sheet of lobe regions that are nearly void of plasma and of a plasma mantle consisting of solar wind plasma penetrating into the magnetosphere. In addition, the overall magnetotail structure was explored by high resolution imaging using a gated optical imager (GOI) and by the subsequent digital analysis of these images. These images confirmed the structure inferred earlier from the magnetic field measurements, which consists of a Y-type magnetic neutral line for northward IMF and the expected X-type structure for southward IMF.

\section{Plasma Transport and Energization in the Magnetotail}

The Earth's magnetotail is a dynamic region of space which has highly extended terrestrial magnetic field lines that are stretched by the interaction with the solar wind as far as a few hundred Earth radii in the anti-sunward direction. Between lobe regions of a nearly uniform earthward or anti-earthward magnetic field, a large reservoir of hot plasma is confined known as the plasma sheet, including a neutral plane where the magnetic field 
reverses direction. This region is referred to as the tail current sheet. When ions travel through the current sheet region in the magnetotail, the fact that the magnetic field is weak and that the field lines are highly curved in this area leads to a breakdown of the particle's adiabatic behavior, and stochastic acceleration can occur in the cross-tail electric field. This nonadiabatic behavior can lead to energization, the formation of ion beams, auroral precipitation at Earth, and many other interesting effects. The global consequences of ion behavior in the Earth's magnetotail field configuration was the main interest of this research topic.

One of the objectives of this work was to understand how the plasma sheet and plasma sheet boundary layer are formed. Observations show that ion distributions in the plasma sheet are more or less isotropic with temperatures of a few keV. The boundary of the plasma sheet, on the other hand, is populated and characterized by ion beams streaming both earthward and tailward along the magnetic field. The source of plasma for both of these regions is ultimately the solar wind, which has a temperature of a few eV and a convection velocity of a few hundred $\mathrm{km} / \mathrm{s}$. The question is as follows: how is this plasma heated to form the plasma sheet and why is the central plasma sheet isotropic, while the boundary layer is populated by energetic field-aligned streaming plasma?

To carry out this study, we used a magnetic field model based on a large satellite magnetometer data set (Tsyganenko 1989), choosing a quiet-time configuration that has an $\mathrm{X}$-line reconnection site at about 60 Earth radii in the tail. Initially a two-dimensional representation of this field model in the north-south plane ( $\mathrm{x}-\mathrm{z}$ plane in GSE coordinates) was used and variations in the dawn-dusk direction ( $\mathrm{y}$ axis) were neglected. A modest crosstail convection electric field of $0.1 \mathrm{mV} / \mathrm{m}$ was imposed along with this magnetic field.

Thousands of ions from different source regions have been launched into this magnetotail field model and their trajectories have been followed. When the ions cross the current-sheet, non-adiabatic behavior occurs and stochastic acceleration results, which heats and energizes the ions. Using virtual detectors placed at different locations in the magnetotail, velocity distribution functions have been constructed in the plasma sheet and the plasma sheet boundary layer, and these results have been compared with observations.

The results show that ions that interact strongly with the current sheet (as determined by the particle's initial energy and pitch angle) are accelerated to high energies and ejected from the current sheet along the boundary of the plasma sheet. These ions form the observed field-aligned beams. Mirror reflection at the Earth creates the tailward directed beam component. At the Earth some of these ions in the loss cone precipitate with a distinct velocity-dispersed structure (VDIS). This VDIS has been observed by satellites poleward of the auroral arc region, at the location predicted by the numerical results found here. 
Ions that do not interact as strongly with the current sheet are not accelerated as much during each interaction, but because these ions mirror bounce in the magnetotail field configuration and convect earthward, they can cross the current sheet many times. Thus, in any given bounce the energy gain is not that much, but because they cross many times, the end result is a large final ion energy. This leads to the formation of the energetic but primarily isotropic plasma sheet located about the neutral plane close to the Earth. The final temperatures of a few $\mathrm{keV}$ found from the numerical results are in good agreement with satellite observations.

\section{Study of Bursty Bulk Flows in the Earth's Central Plasma Sheet}

In a somewhat simplified view, one can distinguish two quite different flow states in the central plasma sheet of the Earth's magnetic tail. The most frequently occurring, most probable state is one of low-velocity, disorderly flow. This state appears to be responsible for circulation of plasma and energy on closed field lines, possibly half the earthward transport of plasma and energy, but a small fraction of the net earthward transport of magnetic flux in the plasma sheet. Statistical studies of the "resting state" of the cross-polar cap electric field suggest that the low velocity plasma sheet flow state might develop a $10-20 \mathrm{kV}$ emf on average. In contrast, bursty bulk flow events (localized highspeed-flow bursts of 5-10 minutes duration) are responsible for most of the earthward magnetic flux transport in the plasma sheet even though any one spacecraft will detect them only a few percent of the time.

The present study concerned the structure and evolution of bursty bulk flow events (BBFs), using the controlled and known separation of the orbits of the two International Sun Earth Explorer satellites (ISEE 1 and 2) and the high time resolution of the Los Alamos plasma instrument (fast plasma analyser, FPE) to time the high speed flows down to the spin period of the satellite (3s). For some events, ISEE 1 and ISEE 2 exhibit significant differences in the magnetic field signatures, despite the fact the two spacecraft were separated by only $0.5 \mathrm{R}_{E}$ in the $\mathrm{z}$-direction. The velocity signatures on the two satellites were also very different: the ISEE 2 velocity in the $x$ direction showed a BBF event whose onset preceded the corresponding ISEE 1 onset by about 20 minutes. This difference can be attributed to the $z$-separation of the satellites. ISEE 1 was closer to the boundary layer and did not sample the high speed flows seen closer to the neutral sheet by ISEE 2. Important differences existed even if ISEE 1 and 2 were both close to the neutral sheet. On one occasion, ISEE 1 sampled a high speed earthward flow, yet ISEE 2 felt the effects of the high speed stream only 5 minutes later. This can be attributed to the $y$-or $z$-separation of the spacecraft. 
From this research, the following view of plasma sheet transport was derived. Empirical models of the plasma sheet magnetic field, because they are adjusted to fit the majority of magnetic field measurements in a given volume of space, correspond to the most probable state of the magnetic field data ensemble, and therefore primarily to the lowvelocity, disorderly flow state. The corresponding slow flow is irregular and inherently time-dependent, and so the ensemble average flow can be consistent (or inconsistent) with the ensemble average magnetic field without either one reflecting an instantaneous flow state. Time dependence may be an essential ingredient, for then a magnetic flux tube defined by a given cross-sectional area in the ionosphere can adjust its volume in the plasma sheet to ensure compliance with the requirement of pressure balance.

This average "quiet" behavior of the plasma sheet is contrasted by short duration dynamic events, consisting of bursty bulk flow events and plasmoids (closed magnetic "bubbles" or rope-like structures within the plasma sheet). These events involve reconfigurations of the plasma sheet magnetic field. Because they are relatively infrequent, the reconfigurations are poorly reflected in the empirical field models. Bursty bulk flows were entirely earthward within $19 R_{E}$, a very small fraction were tailward at ISEE 1 and 2 at 23 $R_{E}$ apogee, and they were mixed earthward and tailward near 30-40 $R_{E}$ (as observed from the satellites IMP 6, 7, and 8), and predominantly tailward in the deep tail (observed by ISEE 3). When they are observed in the near- or mid-tail, fast earthward flows are associated with a northward component of magnetic field, and tailward flows with a southward component consistent with a reconnection source.

In the deep tail, tailward fast flows are very often associated with plasmoids. The durations of plasmoids in the deep tail are comparable with those of bursty bulk flow events. Plasmoids involve reconfigurations of the plasma sheet magnetic field involving $\mathrm{B}_{\mathrm{Z}}$ or $\mathrm{By}_{\mathrm{y}}$ comparable in size to the field strength in the tail lobes, just as BBFs. Plasmoids have the same progression with distance downtail as BBFs; plasmoids are mixed earthward-tailward between $50-100 R_{E}$, and are predominantly tailward beyond $100 R_{E}$. Wherever they are observed, BBFs and plasmoids are often preceded by, or accompanied by, bursts of energetic ions. The energetic ion bursts are observed within one Larmor radius of the plasma sheet boundary layer. All this argues that BBFs, plasmoids, activations of the plasma sheet boundary layer, and energetic particle bursts are created in localized bursty reconnection events, which occur at a variety of distances typically between $30-100 \mathrm{R}_{E}$ downtail.

To the extent that bursty bulk flow events and plasmoids are responsible for the dominant fraction of the central plasma sheet magnetic flux transport, they are the flows visualized in the steady-state reconnection model of the magnetosphere. In short, magneto- 
spheric convection could be the cumulative response to a series of bursty reconnection events both at the magnetopause and in the tail, superposed on an ever-present irregular flow.

\section{Instability Thresholds as Closure Relations in the Earth's Magnetosphere}

Spacecraft observations in the outer magnetosphere typically show that protons consist of several distinct populations: hot $(\mathrm{keV})$ anisotropic protons, very cold $(\mathrm{eV})$ protons that appear to have emerged from the ionosphere, and a warm (tens of $\mathrm{eV}$ ) component (denoted by subscript $w$ ) that is apparently derived from the cold ionospheric protons and which at times exhibits temperature anisotropies $T_{\perp w}>T_{\| w}$ (where $\perp$ and $\|$ correspond to the perpendicular and parallel directions relative to the magnetic field vector) indicative of wave-particle heating. At geosynchronous orbit and greater distances, the hot protons are approximately described by a bi-Maxwellian velocity distribution, and there is substantial observational evidence that the electromagnetic proton cyclotron instability is active in terms of both enhanced magnetic fluctuations and the hot proton anisotropy upper bound described by Equation (1):

$$
\frac{T_{\perp h}}{T_{\| h}}-1=\frac{0.27}{\beta_{\| h}^{0.47}} \quad\left(0.005<n_{h} / n_{e}<0.20\right)
$$

(here the subscript $h$ refers to the hot magnetospheric proton component and $\beta$ represents the ratio between plasma and magnetic pressure). Furthermore, simulations have shown that this instability can heat cold protons to temperatures of order $10 \mathrm{eV}$.

Hybrid simulations investigated the electromagnetic proton cyclotron instability in plasmas with initially cool helium ions and protons (Gary et al, 1996a). They were able to study in detail how helium-ion heating rates varied with several dimensionless parameters including the relative density of the hot protons and the hot proton parallel beta. Gary et al. (1995) carried out further hybrid simulations of the electromagnetic proton cyclotron instability in plasmas with an initially cool proton component. That work not only confirmed the results of earlier simulations, but also showed that the apparent temperature of these warm protons should satisfy

$$
\frac{T_{w}}{T_{\| h}} \cong 0.01\left(\frac{n_{h}}{n_{w}}\right)^{0.9} \quad\left(0.01 \leq n_{h} / n_{w} \leq 0.50\right)
$$


Gary et al (1996b) used Los Alamos geosynchronous plasma data to test this scaling relation and found, for five times at which such anisotropic warm protons were clearly present, that observations were in good agreement with Equation (2), as demonstrated by Figure 1, thereby supporting the hypothesis that these protons are heated by this instability.

\section{Implicit Simulational Studies of the Farley-Buneman Instability in the Equatorial Electrojet}

The Farley-Buneman instability in the equatorial electrojet current system in the Eregion of the ionosphere has been identified as the cause of the observed Type I electron density irregularities. While the linear instability has been understood for nearly three decades, the nonlinear evolution is still unclear. One of the first calculations of this instability in the nonlinear domain was due to Sudan (1983). More recently, Machida and Goertz (1988) have studied the instability numerically with traditional explicit "particle in cell" (PIC) simulation methods. They have calculated electron heating in the polar ionosphere in agreement with Sudan's results, but it is not clear how good or complete the match is. Our goal was to study the instability in the equatorial region, which was ignored by Machida and Goertz. We investigated the cause of saturation, the velocity of the waves in the saturated state (do they travel at the sound speed?) and any other nonlinear phenomena. The significance of this work derives from the large number of unanswered questions about Type I waves. These include, among others, the observation of vertically propagating twostream waves (perpendicular to the current), the constant phase velocity of type I waves at any angle with respect to the current, and the details of the observed wave number spectra (Kelley 1989). Much of the detailed observational data about these waves was -- and still is - gathered by Cornell University researchers. This gave us the ability to closely correlate our results with the experimental data.

We originally planned to evaluate the nonlinear behavior of the Farley Buneman Instability solely with the Los Alamos ANTHEM implicit plasma code. This model can treat electron kinetic influences on the instability's long-time-scale evolution. However, our collaborator Meers Oppenheim (Cornell) has developed two newer models, which allow for speedier electron fluidic treatments. The first is a two-fluid simulation that also incorporates linear ion kinetic damping. This enabled us to evaluate quickly the wave behavior assuming that kinetic effects are negligible. The second is an explicit particle code that uses particle ions and fluid electrons. This enabled us to determine whether ion kinetic effects are substantially modifying the ion fluid behavior.

Initial results of the fluid simulation showed three nonlinear effects: (1) saturation, (2) nonlinear rotation of the wave direction away from strictly horizontal, and (3) nonlinear coupling from the predominant horizontal wave structure of wavelength $1.5 \mathrm{~m}$ to a long 
wavelength vertical wave. The first two effects have been confirmed by the PIC code. The third effect is also an interesting result because the observational database also shows these, as yet unexplained, waves.

Using the new models we explored typical conditions for the daytime equatorial electrojet at an altitude of $100 \mathrm{~km}$ to $105 \mathrm{~km}$. The simulations produce a "typical" saturated state for the FB waves. From the calculations we estimate a phase velocity for the waves of $430 \mathrm{~m} / \mathrm{s}$, which compares to $600 \mathrm{~m} / \mathrm{s}$ predicted by the linear theory. The acoustic speed is $c_{\mathrm{s}}=340 \mathrm{~m} / \mathrm{s}$. The simulations provided evidence that ion Landau damping may not play an important role in eliminating short wavelength modes. After saturation, the distribution of ions in velocity space differs from the initial Gaussian profile only by an offset of its mean velocity by the ion Pederson drift velocity. This implies that a fluid model for ion behavior may be adequate. The studies also revealed the presence of small-scale waves riding along the crests of the principal waves, traveling both upward and downward.

These are nonlinear $\mathbf{E} \times \mathbf{B}$ effects with the electric field $\mathbf{E}$ arising from the field of the principal waves. These may be responsible for the turning of the waves off the direction of the drift velocity.

\section{Collective Processes in Cosmic Dusty Plasmas}

Dusty plasmas occur in many space and astronomical environments, among them planetary atmospheres and rings, asteroid zones, comets, protostellar and protoplanetary clouds, supernova remnants, the interstellar medium and the Earth's ionosphere and magnetosphere. The goal of this task was to investigate waves and instabilities in dusty magnetized plasmas and to apply the results to various cosmic environments, as well as to laboratory plasmas where applicable. Extending our theory of secondary electron emission from small grains and their effect on cosmic and laboratory plasmas, we have studied several dusty plasma instabilities and their role in planetary rings, and considered the dynamics of dust grains in quasi-equilibrium traps.

The presence of dust in a plasma leads to new sources of free energy that can drive plasma instabilities. We have investigated one such instability that can occur in the rings of Saturn. In the inner magnetosphere of Saturn the plasma rotates with the planet, while the dust grain orbital motion is determined by gravity. This leads to a relative drift between the dust grains and the plasma; the relative drift can exceed the ion thermal velocity and excite an ion acoustic instability.

We have modeled the development and evolution of the instability with a particle-incell code, in which both the dust and plasma ions are treated as particles and the electrons 
are assumed to have a Boltzmann distribution. The growth rate of the instability is roughly the dust plasma frequency and the wavelength is the Debye length. The instability grows rapidly to a relatively low level of fluctuations (fluctuating potential energy comparable to the ion temperature). The plasma ions at small positive velocities are affected most, as some of these ions become trapped by the growing waves, which eventually causes the wave growth to saturate. The dust ions, on the other hand, are merely modulated by the waves on this time scale, although when averaged over space, it appears as strong heating of the dust. The fact that the relative dust-plasma ion drift speed increases with distance from the planet suggests that stronger plasma heating should occur at larger radius, which is consistent with measurements made by the Voyager spacecraft. The presence of the instability also helps explain the relatively isotropic velocity distribution of the plasma ions, which in the absence of some sort of scattering process should be more ring-like in nature.

\section{DISCLAIMER}

This report was prepared as an account of work sponsored by an agency of the United States Government. Neither the United States Government nor any agency thereof, nor any of their employees, makes any warranty, express or implied, or assumes any legal liability or responsibility for the accuracy, completeness, or usefulness of any information, apparatus, product, or process disclosed, or represents that its use would not infringe privately owned rights. Reference herein to any specific commercial product, process, or service by trade name, trademark, manufacturer, or otherwise does not necessarily constitute or imply its endorsement, recommendation, or favoring by the United States Government or any agency thereof. The views and opinions of authors expressed berein do not necessarily state or reflect those of the United States Government or any agency thereof. 


\section{Publications}

Angelopoulos, V., C. F. Kennel, F. V. Coroniti, R. Pellat, H. E. Spence, M. G. Kivelson, R. J. Walker, W. Baumjohann, W. C. Feldman, J. T. Gosling, and C. T. Russell, Characteristics of ion flow in the quiet state of the inner plasma sheet, Geophys. Res. Lett. (1993).

Ashour-Abdalla, M., R. L. Richard, D. Schriver, L. Zelenyi, and J. Raeder, Understanding the Earth's distant magnetotail, Adv. Space Res. (1993).

Ashour-Abdalla, M., D. Schriver, L. M. Zelenyi, J. M. Bosqued, F. V. Coroniti, Mechanisms of particle precipitation: A controversial issue, Physics of Space Plasmas (1992), SPI Conference Proceedings and Reprint Series, No. 12 (1993).

Birn, J., and M. Hesse, Influence of plasma sheet thickening toward the tail flanks on magnetotail stability and dynamics, J. Geophys. Res., 99, 5847, 1994.

Birn, J., K. Schindler, L. Janicke, and M. Hesse, Magnetotail dynamics under isobaric constraints, J. Geophys. Res., 99, 14,863, 1994.

Birn, J., K. Schindler, and M. Hesse, Magnetotail dynamics: MHD simulations of driven and spontaneous dynamic changes, in Substorms 2, Proc. 2nd Int. Conf. on Substorms, Fairbanks, Alaska, 7-11 March 1994, submitted, 1994.

Birn, J., S. Peter Gary, and Michael Hesse, Microscale anisotropy reduction and macroscale dynamics of the magnetotail, J. Geophys. Res., 100, 19,211, 1995.

Birn, J., Michael Hesse, and S. Peter Gary, The role of microprocesses in macroscale magnetotail dynamics, in Cross-Scale Coupling in Space Plasmas, Geophys. Monogr. Ser., vol. 93, edited by J. L. Horwitz, N. Singh, and J. L. Burch, p. 275-285, AGU, Washington, D.C., 1995.

Birn, J., Magnetotail stability and dynamics: Progress 1991-1993, Surveys in Geophysics, 16, 299-330, 1995.

Chow, V. W., D. A. Mendis, and M. Rosenberg, Secondary emission from small dust grains at high electron energies, IEEE Trans. Plasma Sci., 22, 179, 1994.

Chow, V.W. and M. Rosenberg, Electrostatic ion cyclotron instability in dusty plasmas, Planet. Space Sci., 43, 613-618, 1995.

Chow, V.W. and M. Rosenberg, Electrostatic ion cyclotron instabilities in negative ion plasmas, submitted to Phys. Plasmas, 1995.

Convery, P. D., and S. P. Gary, Electromagnetic proton cyclotron ring instability: Threshold and saturation, J. Geophys. Res., submitted, 1996.

Elphic, R. C., Observations of flux transfer events; A review, in Physics of the Magnetopause, edited by P. Song, B. U. Ö. Sonnerup, and M. F. Thomsen, pp. 225-233, American Geophysical Union, Washington, DC, 1995. 
Gary, S. P., M. F. Thomsen, L. Yin, and D. Winske, Electromagnetic proton cyclotron instability: interactions with magnetospheric protons, J. Geophys. Res., 100, 21961, 1995.

Gary, S. P., L. Yin, and D. Winske, Electromagnetic proton cyclotron instability: heating of cool magnetospheric helium ions, Annales Geophysicae, 14, 1, 1996a.

Gary, S. P., V. M. Vazquez, and D. Winske, Electromagnetic proton cyclotron instability: Proton velocity distributions, J. Geophys. Res., 101, 13,327, 1996 b.

Hesse, M., J. Birn, D. N. Baker, and J. A. Slavin, MHD simulations of the transition of magnetic reconnection from closed to open field lines, J. Geophys. Res., 101, $10,805,1996$.

Hesse, Michael, Dan Winske, Masha Kuznetsova, Joachim Birn, and Karl Schindler, Hybrid modeling of the formation of thin current sheets in magnetotail configurations, $J$. Geomagn. Geoelec., 120, 749 (1996).

Karimabadi, H., D. Krauss-Varban, N. Omidi, S. A. Fuselier, and M. Neugebauer, Low frequency instabilities and the resulting velocity distributions of pickup ions at comet Halley, J. Geophys. Res., 99, 21541-21556 (1994).

Karimabadi, H., N. Omidi, and S.P. Gary, Ion scattering and acceleration by low frequency waves in the cometary environment, in Solar System Plasmas in Space and Time, edited by J. L. Burch and J. H. Waite, Geophys. Monogr. Ser., 84, 71-84, AGU, Washington DC, 1994.

Karimabadi, H., D. Krauss-Varban, and N. Omidi, Kinetic structure of intermediate shocks: Implications for the magnetopause, J. Geophys. Res., 100, 11957-11979 (1995).

Krauss-Varban, D., Structure and length scales of rotational discontinuities, J. Geophys. Res., 98, 3907, 1993.

Krauss-Varban, D., N. Omidi, and K. B. Quest, Mode properties of low frequency waves: Kinetic theory versus Hall-MHD, J. Geophys. Res., 99, 5987, 1994.

Krauss-Varban, H. Karimabadi, and N. Omidi, Kinetic structure of rotational discontinuities: Implications for the magnetopause, J. Geophys. Res., 100, 11981-11999 (1995).

Kuo, H., C.T. Russell, and G. Le, Statistical studies of flux transfer events, J. Geophys. Res, 100, 3513-3519, 1995.

Le, G., C. T. Russell and H. Kuo, Flux transfer events: Spontaneous or driven? Geophys. Res. Lett., 20, 791-794, 1993.

Le, G., C.T. Russell, and J.T. Gosling, Structure of the magnetopause for low Mach number and strongly northward interplanetary magnetic field, J. Geophys. Res, 99, 23,723-23,734, 1994.

Le, G., C.T. Russell, J.T. Gosling, and M.T. Thomsen, ISEE observations of the low latitude boundary layer for northward interplanetary magnetic field: Implications for cusp reconnection, J. Geophys. Res., 1995. 
Lindsay, G. M., J. G. Luhmann, C. T. Russell, and J. T. Gosling, CME Characteristics in the inner heliosphere: Relationship to corona velocity, submitted to J. Geophys. Res., 1995.

Maravilla, D., K. R. Flammer, and D. A. Mendis, On the injection of fine dust from the Jovian magnetosphere, Astrophys. J., 438, 968-974, 1995.

Maravilla, D., K. R. Flammer, and D. A. Mendis, The nature of the orbits of charged dust injected into the Jovian magnetosphere during the tidal break-up of comet ShoemakerLevy 9, Astrophys. Space Sci., in press, 1995.

Mason, R. J., ANTHEM: User's Manual--Edition 1.1, Los Alamos Report LA-UR 93888, March 5, 1993.

Mason, R. J., R. N. Sudan, P. Auer, C. Seyler, B. Oliver, J. Greenly, and L. Adler, Nonlinear magnetic field transport in opening switch plasmas, Phys. Fluids $B$, vol. 5, $1115,1993$.

McKean, M. E., N. Omidi, and D. Krauss-Varban, Wave and ion evolution downstream of quasi-perpendicular bow shocks, J. Geophys. Res., 100, 3427, 1995.

Mendis, D.A. and M. Rosenberg, Cosmic dusty plasma, Ann. Rev. Astron. Astrophys., 32, 419, 1994.

Omidi, N., and H. Karimabadi, Nonlinear evolution of ULF waves and their transition to turbulence, $A G U$ monograph, Proceedings of the Yosemite Meeting on Solar System Plasma Physics: Resolution of Processes in Space and Time, submitted, 1993.

Omidi, N., A. O'Farrell, D. Krauss-Varban, Sources of magnetosheath waves and turbulence, Adv. Space Res., in press, 1993.

Omidi, N., and D. Winske, Structure of the magnetopause as inferred from the kinetic Riemann problem, J. Geophys. Res., submitted, 1994.

Omidi, N., and D. Winske, Kinetic structure of the magnetopause as inferred from 1-D hybrid simulations, J. Geophys. Res., 100, 11,935, 1995.

Rosenberg, M. and N. A. Krall, High frequency drift instabilities in a dusty plasma, Planet. Space Sci., 42, 889-894, 1994.

Rosenberg, M. and N.A. Krall, Modified two-stream instabilities in dusty space plasmas, Planet. Space Sci., 43, 619-624, 1995.

Rosenberg, M., D. A. Mendis, and V. W. Chow, Weakly ionized cosmic gas: ionization and characterization, Astrophys. Space Sci., 222, 247-253, 1994.

Rosenberg, M. and D. A. Mendis, UV-induced Coulomb crystallization in a dusty gas, IEEE Trans. PlasmaSci., 23, 177-179, 1995.

Rosenberg, M. and N. A. Krall, Low frequency drift instabilities in a dusty plasma, submitted to Phys. Plasmas, 1995.

Russell, C. T., G. Le, and H. Kuo, The occurrence rate of flux transfer events, $A d v$. Space Res., in press, 1994. 
Russell, C. T., A Study of flux transfer events at different planets, Adv. Space Res., 16, (4)159-(4)163, 1995.

Russell, C. T., The structure of the magnetopause, in Physics of the Magnetopause, 8198, American Geophysical Union, Washington, DC, 1995.

Scurry, L., C.T. Russell, and J.T. Gosling, A statistical study of accelerated flow at the dayside magnetopause, J. Geophys. Res, 99, 14,815- 14,829, 1994.

Song, P., G. Le, and C. T. Russell, Observational differences between flux transfer events and surface waves at the magnetopause, J. Geophys. Res., 99, 2309-2320, 1994.

Walthour, D. W., B.U. Ö. Sonnerup, R.C. Elphic, and C.T. Russell, Double vision: Remote sensing of a flux transfer event with ISEE 1 and 2, J. Geophys. Res., 99, 85558563, 1994.

Winske, D., and M. E. Jones, Forces on particulates in rf discharges, IEEE Trans Plasma Sci., 22, in press, 1994.

Winske, D., and N. Omidi, Diffusion at the magnetopause: Hybrid simulations, J. Geophys. Res., 100, 11,923 (1995).

Winske, D., V. A. Thomas, and N. Omidi, Diffusion at the magnetopause: A theoretical perspective, in Physics of the Magnetopause, edited by P. Song, B. U. O. Sonnerup, and M. F. Thomsen, Amer. Geophys. Union, p. 321, 1995.

Winske, D., S. P. Gary, M. E. Jones, M. Rosenberg, V.W. Chow, and D.A. Mendis, Ion heating in a dusty plasma due to the dust/ion acoustic instability, Geophys. Res. Lett, $21,2069,1995$.

Yur, G., H. U. Rahman, J. Birn, F. J. Wessel, and S. Minami, An improved simulation facility for magnetospheric modelling, J. Geophys. Res., in press, 1995.

Yur, G., H. U. Rahman, J. Birn, F. J. Wessel, and S. Minami, Magnetotail structures in a laboratory magnetosphere, J. Geophys. Res., submitted, 1995. 


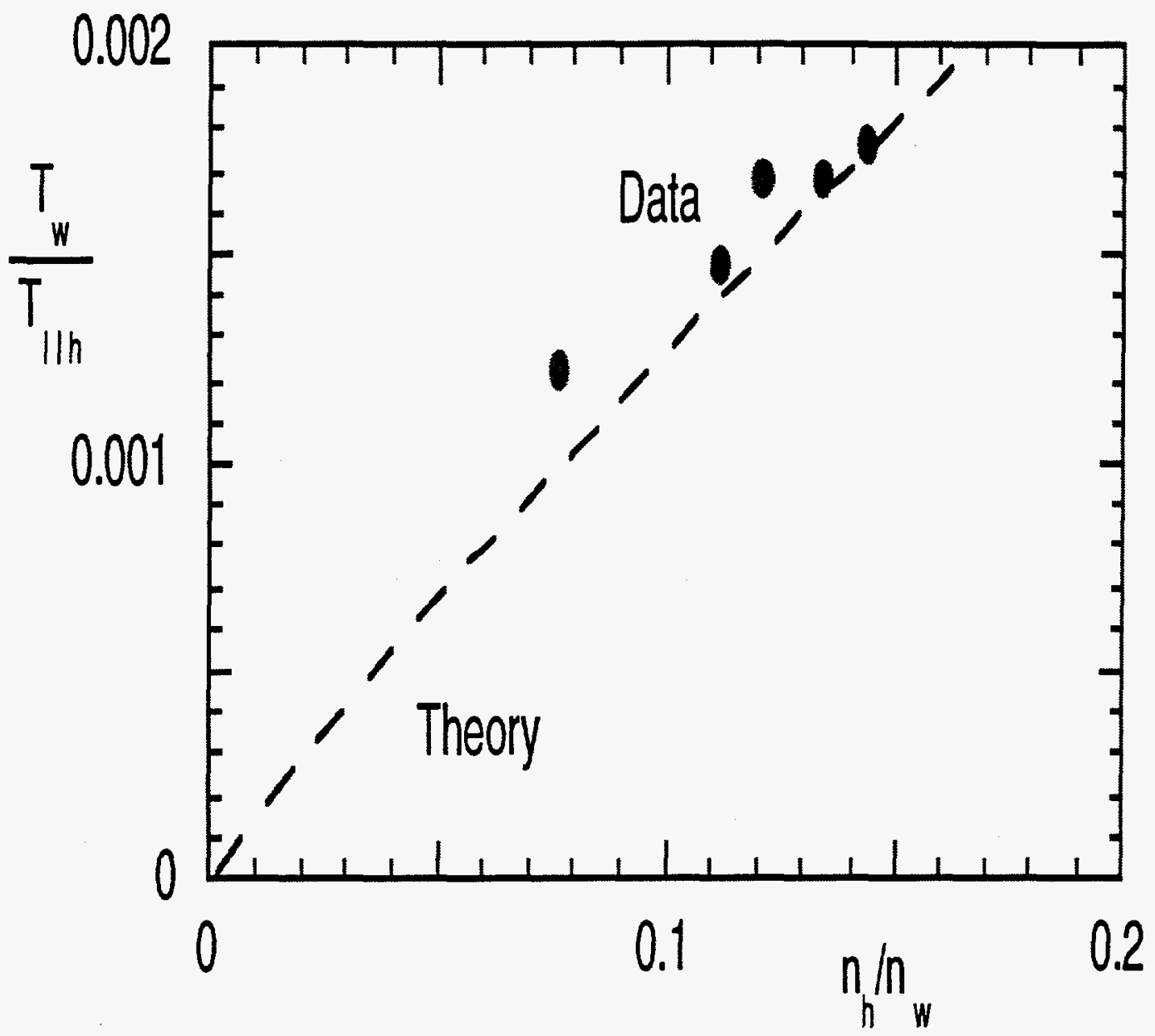

Figure 1. The dimensionless warm proton temperature as a function of the hot proton/warm proton density ratio. The dashed line represents the theoretical/simulation scaling of Equation (2). The solid dots represent five observations from Los Alamos instrumentation on geosynchronous spacecraft 1989-046 on 27 June 1990, 28 June 1990, and 23 January 1994. From Gary et al. (1996b). 